\title{
Cortically Induced Presynaptic Inhibition in Cat's Lateral Geniculate Body
}

\author{
By \\ Hisao Suzuki and Eiichi Kato \\ From the Institute of Brain Diseases, Tohoku University \\ School of Medicine, Sendai \\ (Received for publication, April 18, 1965)
}

\begin{abstract}
The effect of cortical stimulation on the evoked response of the lateral geniculate body (LGB) was studied in the cat locally anesthetized and immobilized with Flaxedil. The evoked response of the LGB induced by single shock stimulation of the optic tract consisted of pre- and postsynaptic components. When single shock stimulation was applied to the visual cortex $50-100$ msec prior to optic tract stimulation, the postsynaptic component of the evoked response decreased in amplitude, while the presynaptic component either remained unaltered or was slightly reduced. Brief repetitive stimulation of the visual cortex produced a significant reduction of the amplitude of the presynaptic component. When the excitability of the optic tract fibers in the LGB was tested with an antidromic action potential of the optic tract as index, it was found to be enhanced in the phase in which the presynaptic component of the evoked response was reduced. These results suggest that cortical stimulation may induce presynaptic depolarization which causes inhibition of nervous transmission in the LGB (presynaptic inhibition).
\end{abstract}

Volleys in the optic nerve set up by electrical stimulation give rise to the evoked response in the lateral geniculate body (LGB) and this response consists of the presynaptic and postsynaptic components. ${ }^{4}$ When a conditioning stimulus is applied to the striate or parastriate cortex, the postsynaptic component suffers remarkable depression lasting as long as $500 \mathrm{msec}$, whereas the presynaptic component undergoes only a little change. Therefore, it may be suggested that the depression is generated by a postsynaptic process in the LGB. ${ }^{10}$ However, slight depression occurs occasionally in the presynaptic component, too, indicating that the effect of cortical stimulation also involves a presynaptic process. Furthermore, the depression of the presynaptic component becomes manifest when a brief tetanic stimulus is used instead of a single conditioning stimulus. In the present investigation, the cortically induced depression of the presynaptic component of the geniculate response is analyzed to elucidate its mechanism. 


\section{METHODS}

Cats were used exclusively. Under ether anesthesia, the trachea was cannulated and the anesthesia was maintained during the following operative procedure. The animal's head was fixed to a stereotaxic apparatus, the dorsal surface of the cortex was exposed widely and the posterior cistern was opened. The corneae of both eyes were exposed to take out the lenses and the vitreous body fluid. Then the optic discs of the eyes were cauterized under direct observation by means of 'Hyfrecator' (the Birtcher Corporation). Thus, the pathway between the retina and the LGB was blocked completely. All pressure points and wound margins of the animal were infiltrated with procaine to get local anesthesia because during the experimental procedure no ether anesthesia was used. The animal was immobilized with repeated injection of Flaxedil and respirated artificially.

Bipolar stimulating electrodes were inserted stereotaxically into the optic tract to induce the evoked response in the LGB. The electrodes used consisted of two $100 \mu$ insulated silver wires with bare tips lacquered together so that tip separation was $1-3 \mathrm{~mm}$. Since the $100 \mu$ silver wires were too flexible to be inserted into the brain tissue, they were cemented to a $300 \mu$ stainless steel tube with Insul-X so that the tip of the attached silver wires was flush with that of the tube. The assembly, two silver wires and a stainless steel tube, was stiff enough to be poked straight down into the brain tissue. Recording of the evoked response in the LGB was made with the same type of the electrodes as for stimulating the optic tract.

The method of testing excitability changes in the fiber terminals of the optic tract was similar to that described by Wall and Johnson ${ }^{18}$ and also that applied by Eccles et al. ${ }^{8}$ to the spinal cord and the relay nuclei in the brain stem ${ }^{2}$ : A stimulus was applied through the bipolar electrodes inserted into the LGB to excite the optic tract fibers close to synaptic terminals in the LGB, while recording was made with the same type of electrodes in the optic tract. Excitability changes of the stimulated site in the $L G B$ resulted in changes in size of the antidromically conducting response of the optic tract.

For application of a conditioning stimulus to the visual cortex and its surrounding areas, an insulated silver wire with a bare tip of $500 \mu$ was used and negative pulses were applied to the cortical surface through this electrode. An indifferent electrode, a silver plate wrapped by Ringer-soaked cotton, was placed at the margin of the opened skull.

The response taken with these electrodes was displayed on a dual beam oscilloscope through an a.c. coupled amplifier with time constant of $0.03 \mathrm{sec}$. An interval between two stimuli, for example the conditioning stimulus to the striate cortex and the test stimulus to the optic tract for eliciting the evoked response in the LGB, was measured precisely by use of an electronic digital counter.

After each experiment, the brain was taken out to harden in 10 per cent 
formalin, and frozen sections were made to identify tracks of the electrodes.

\section{RESULTS}

Effect of repetitive cortical stimulation on evoled response in the $L G B$

When the recording electrodes were in an adequate locus of the LGB, a typical evoked response such as described by Bishop and MeLeod was obtained by a single stimulus applied to the ipsilateral optic tract. ${ }^{4}$ Records illustrated in Fig. 1 were taken from a point corresponding to $\mathrm{F} 6, \mathrm{~L} 9, \mathrm{H} 4$ of the stereotaxic atlases while a single shock stimulus was applied at a point corresponding to $\mathrm{F} 12, \mathrm{~L} 5, \mathrm{H}-5$. The response occurred immediately after a shock artefact and was a positive-negative diphasic spike of about 0.5 msec duration (presynaptic component), which was followed by a negative spike of 1 msec duration (postsynaptic component). Besides these dominant spikes, additional small negative spikes are seen before and after the postsynaptic component, and they may represent activities of the slow conducting system in the optic tract (comparable to Bishop's $t_{2}$ and $r_{2}$ ).

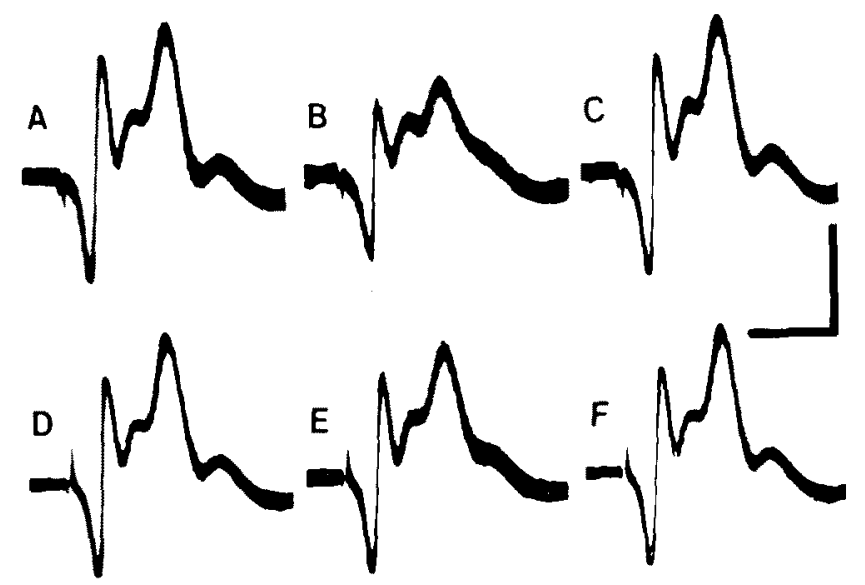

Fig. 1. Effect of cortical stimulation on geniculate evoked response. Each response was obtained by single stimulus to ipsilateral optic tract. A, control response. B, response conditioned by train of stimuli ( 5 at $300 / \mathrm{sec}$ ) applied to ipsilateral visual cortex 80 msec earlier. C, control response taken after B. D and F, control responses. E, response conditioned by train of stimuli of same parameters as in B applied to contralateral visual cortex. For all records illustrated in this figure 7 sweeps were superposed. Vertical bar, $500 \mu \mathrm{V}$. Horizontal bar, $1 \mathrm{msec}$. Upward deflection denotes negativity at deeper tip of electrodes.

When conditioned by a brief tetanic stimulus to the ipsilateral visual cortex, both presynaptic and postsynaptic components of the evoked response in the LGB showed a prolonged depression. In Fig. 1B, a train of five rectangular pulses at $300 / \mathrm{sec}$ was delivered to the surface of the ipsilateral visual cortex corresponding to 
the stereotaxic coordinate of $\mathrm{F} 4, \mathrm{~L} 2,80 \mathrm{msec}$ prior to the test optic tract stimulus. It was clearly seen that the presynaptic component together with the postsynaptic component was reduced considerably in amplitude. This cortically induced depression of the geniculate response was well reversible: Without the conditioning stimulus to the visual cortex a geniculate response of the control size was obtained again (Fig. 1C). In D, E and F, a series of similar experiments was made in one and the same animal using a conditioning stimulus to the contralateral instead of ipsilateral visual cortex. In this case, the stimulus induced only a small effect on the presynaptic component, although the postsynaptic component showed a considerable amount of depression (Fig. 1E). In some experiments, however, stimulation of contralateral visual cortex induced depression of the presynaptic component much clearer than that illustrated in Fig. 1.

Effect of repetitive cortical stimulation on excitability of optic nerve terminals in the $L G B$

The bipolar electrodes, inserted into the LGB, were moved up and down to find out a position where the typical geniculate response of Bishop and McLeod was picked up. At such a position stimulation through the electrodes may excite optic tract fibers close to their terminals in the LGB. When the optic tract fibers was excited in such a way, the antidromic conducting action potential along the fibers could be recorded from the electrodes inserted into the ipsilateral optic tract. Though the form of the antidromic response changes according to the position of the recording electrodes, the response consisted of two distinct components in most cases. In Fig. 2 antidromic action potentials of positive-negative biphasic spikes (action potentials) are illustrated. The conduction velocities of the two spikes computed
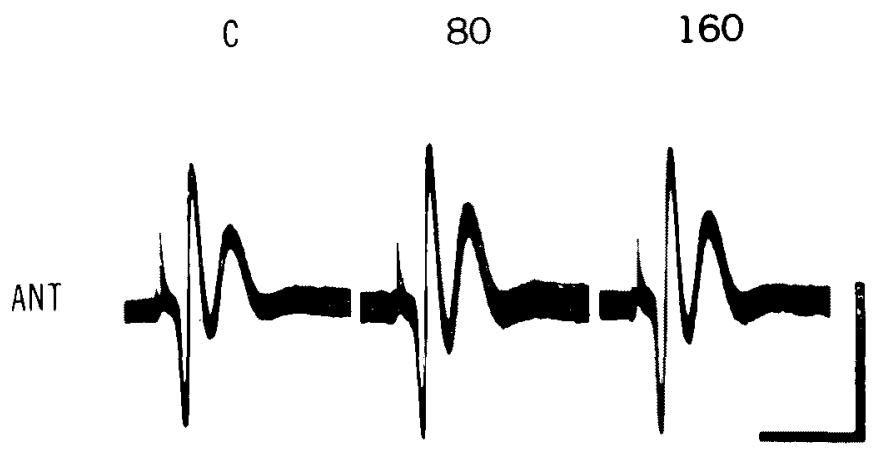

Fig. 2. Effect of cortical stimulation on antidromic artion potential of optic tract. Antidromic response was evoked by stimulus applied to ipsilateral LGB. $\mathrm{C}$, control response. Other two records were preceded by brief tetanic stimulation (5 at $300 / \mathrm{sec})$ to ipsilateral visual cortex by intervals indicated in msec. Vertical bar, $500 \mu \mathrm{V}$. Horizontal bar, 1 msec. 
by dividing the distance between stimulating and recording sites by peak negative latencies were $35 \mathrm{~m} / \mathrm{sec}$ and $16 \mathrm{~m} / \mathrm{sec}$ respectively, values comparable to those obtained by previous authors. ${ }^{3}$ In the middle and right records of Fig. 2 , conditioning was made by brief repetitive stimulation ( 5 shocks at $300 / \mathrm{sec}$ ) of the ipsilateral visual cortex 80 and $160 \mathrm{msec}$ earlier than the testing stimulus to the LGB respectively. As can be seen clearly in both records, the amplitudes of the fast and slow conducting action potentials increased to 130 per cent of the control. This fact indicates that the conditioning stimulus to the visual cortex induces some direct effect upon the optic tract fibers. The effect may be based on the depolarization of the fiber terminals which is induced by conditioning stimulation to the visual cortex; the number of the fibers responding to the test stimulus applied to the LGB would increase under such a depolarized state of the fibers. This may be the reason why the amplitude of the antidromic action potential is increased by the conditioning stimulus. The following experiments will support this explanation.

Time course of cortical effects

In Fig. 3 are given the full time courses of the cortically induced depression of

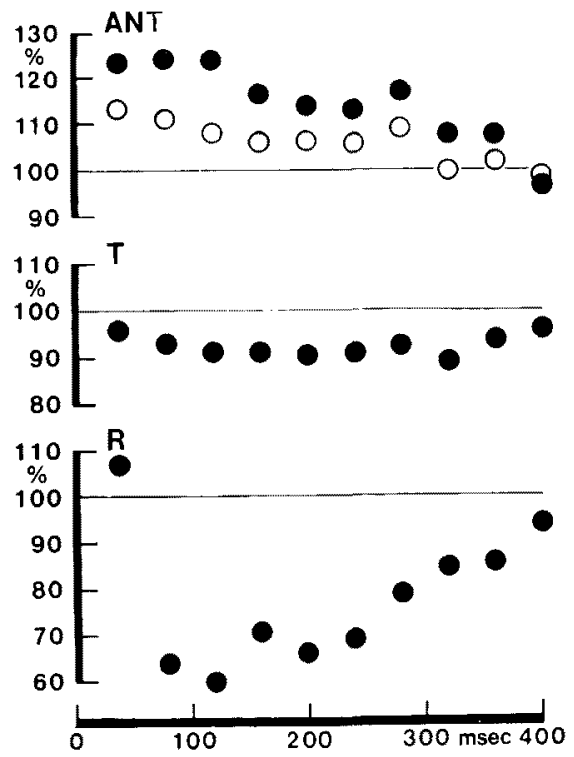

Fig. 3. Time courses of cortically induced depresssion of geniculate evoked response and concurrently induced increase of antidromic action potential at optic tract. As conditioning stimulus, train of 5 shocks at $300 / \mathrm{sec}$ was applied to ipsilateral visual cortex. Ordinate gives size of conditioned response as percentage of control. ANT, time courses of antidromic action potentials. $T$ and $R$, those of presynaptic and postsynaptic components of geniculate evoked response. Abscissa gives conditioning-testing interval. 
the orthodromic response in the LGB and that of simultaneous increases in size of the antidromically conducting action potentials along the optic tract. In the uppermost graph of this figure, sizes of the fast (open circles) and slow (filled circles) antidromic action potentials were plotted as percentage of the control against the conditioning-testing intervals. Since the antidromic action potential showed positive-negative diphasic configuration, full amplitude from positive to negative peaks was measured as a size of the action potential. As seen in this graph, the slow conducting action potential was much increased at about $100 \mathrm{msec}$ after the conditioning stimulus. Recovery was attained over $400 \mathrm{msec}$. The fast conducting action potential exhibited a similar time course of the increase in size which was usually less than that of the slow action potential (see open circles in the uppermost graph).

Depression of the presynaptic component of the geniculate response is illustrated in the middle graph. The maximal depression of the component was at 100-300 msec in this graph. The postsynaptic component showed also prolonged depression by cortical stimulation as seen in the lowest graph. The depression was almost complete at $400 \mathrm{msec}$.

Fig. 4 illustrates another example of the time course of the cortically induced increase in the antidromic action potential whose conduction velocity was $17 \mathrm{~m} / \mathrm{sec}$. In graph $A$, the strength of the test stimulus for generating the antidromic action potential was four times the threshold, and in graph B two times the threshold. When tested by a shock as strong as four times the threshold, condi-

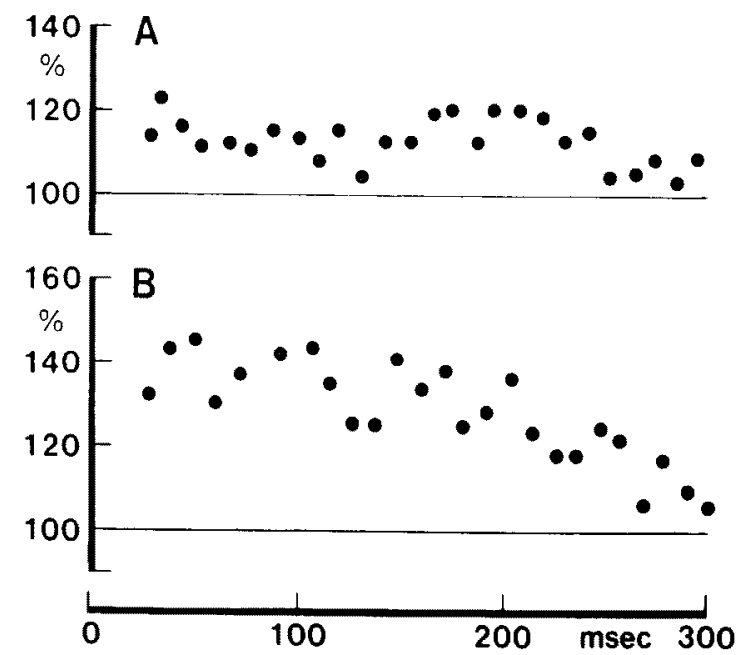

Fig. 4. Time courses of cortically induced increases 7 inl antidromic action potential. A, experiment with stronger test stimulus (4 times threshold for evoking antidromic response). B, exporimont with wakker test stimulus (2 times threshold). Note that effect is clearer in $\mathrm{B}$ than in $\mathrm{A}$. 
tioning by brief tetanic stimulation of the ipsilateral visual cortex caused a slight increase in the antidromic action potential by only 20 per cent. The effect of cortical conditioning was more obvious in the antidromic action potential evoked by a weak stimulus such as two times threshold; an increase as much as 40 per cent of the control was obtained in this case.

Sizes of antidromic and orthodromic responses and cortically induced effects

In the previous section, it was suggested that the cortically induced increase could be obtained prominently in the antidromic action potential of submaximal size. Therefore, more systematic investigation was made as to the dependence of the cortically induced effect on the size of the antidromic action potential. In Figs. 5B and 5D, filled circles show the size of the fast and slow antidromic action potentials against the strength of the test stimulus while open circles show the antidromic action potentials conditioned by a repetitive cortical stimulus $50 \mathrm{msec}$ prior to the test stimulus. $\mathrm{A}$ and $\mathrm{C}$ illustrate increments of the conditioned
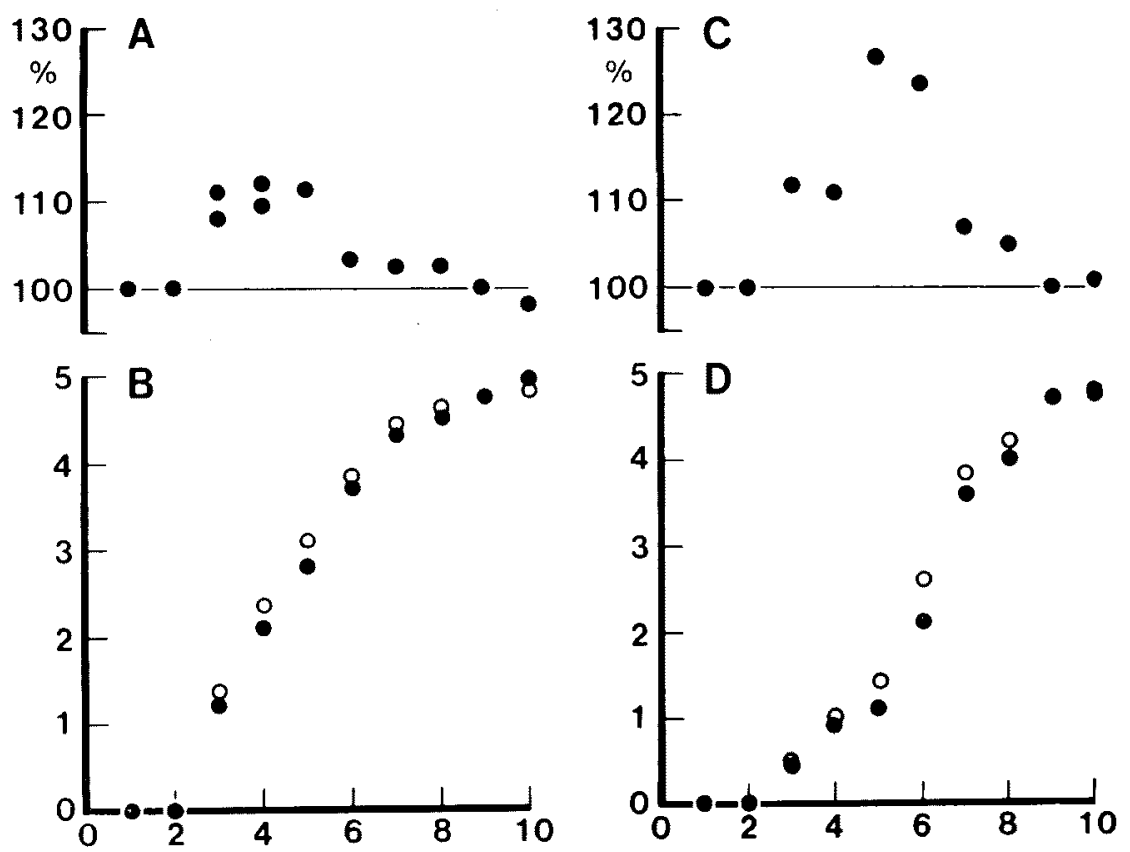

Fig. 5. Size of antidromic action potential of optic tract and cortically induced augmentation. In $\mathrm{B}$, sizes of fast antidromic action potentials were plotted in arbitrary unit against stimuli of various strengths to LGB. Filled circles in B, action potentials evoked by test stimuli alone. Open circles, those conditioned by cortical stimulation (10 shocks at $300 / \mathrm{sec}$ ). A, percentage increment of action potential. D, size of slow conducting action potential vs. various strengths of stimuli to LGB. C, percentage increment of slow action potentials by cortical stimulation. 

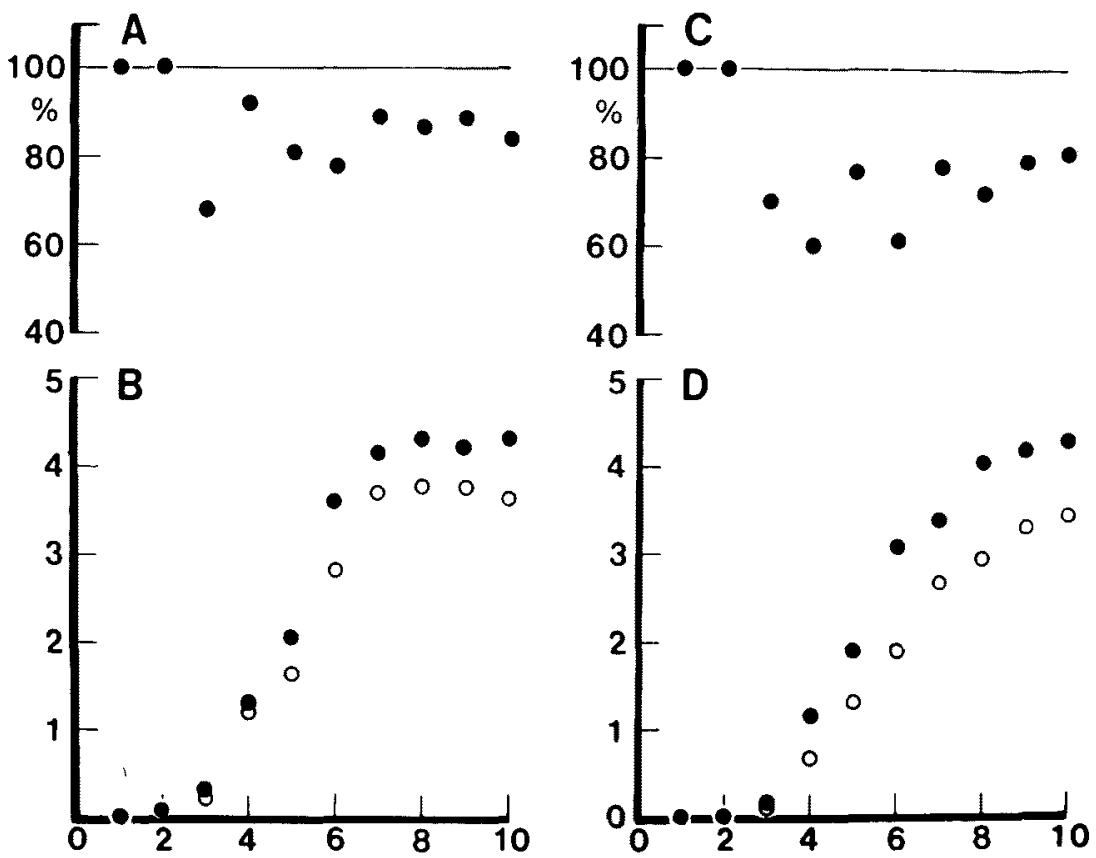

Fig. 6. Size of geniculate evoked response and cortically evoked depression. In B, sizes of presynaptic components are plotted in arbitrary unit against optic tract stimuli of various strengths. Filled circles, presynaptic components evoked by test stimuli alone. Open circles, conditioned by cortical stimulation (10 shocks at $300 / \mathrm{sec}$ ). In A, presynaptic component is shown as percentage of control. $\mathrm{D}$, size of postsynaptic component vs. various strengths of optic tract stimuli. $\mathrm{C}$, expressed in percentage of control.

antidromic action potentials as percentage of the control. As shown in these graphs, the maximal increment was obtained when the antidromic action potential was about half of the maximal size and the increment was almost null when the size of the antidromic action potential was maximal. This finding suggests that the increase of the antidromic action potential is due to the increase in number of fibers excited because the effect can be seen only for submaximal test stimulus; in the maximal action potential there is no additional fibers to be excited.

In Figs. $6 \mathrm{~B}$ and $6 \mathrm{D}$, filled circles refer to sizes of the presynaptic and postsynaptic components respectively of the geniculate response evoked by stimuli of various intensities applied to the ipsilateral optic tract. On the other hand, open circles of these graphs represent their sizes conditioned by brief tetanic stimulation of the ipsilateral visual cortex. From these data, reduction of both components was obtained and represented in $\mathrm{A}$ and $\mathrm{C}$ as percentage of the control. As can be seen in $A$ and $C$, the cortically induced depression was observable even when the size of the response was maximal, and this was the most striking difference from the 
cortically induced effect on the antidromic action potential mentioned above. The little dependence of the cortically induced depression on the size of the orthodromic evoked response may be accounted for in terms of the size of the action potential of each presynaptic fiber better than in terms of the number of fibers excited; reduction of action potential amplitude under depolarization is a well-known neurophysiological fact. Therefore, it is sufficient to assume that the conditioning cortical stimulus induces depolarization in the terminals of optic tract fibers.

\section{Number of conditioning stimuli and their effect}

Fig. 7 shows antidromic action potentials at the optic tract conditioned by various numbers of stimuli to the visual cortex. The repetition rate and conditioning-testing interval were held constant ( $300 / \mathrm{sec}$ and $80 \mathrm{msec}$, respectively). As can be clearly seen in the records on the right side and their plotted graph on the left, the antidromic action potential increased in amplitude almost linearly with the number of the conditioning stimuli.
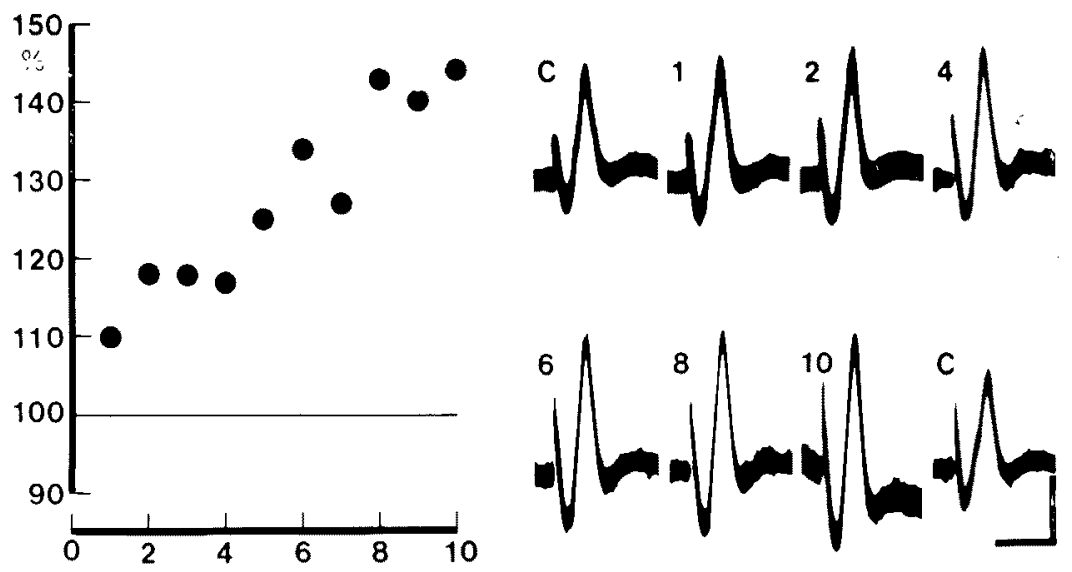

Fig. 7. Cortically induced augmentation of antidromic action potential at optic tract and number of stimuli to ipsilateral visual cortex. Parameters of conditioning stimulus to visual cortex were changed so that with frequency of repetition, duration and strength of each shock all fixed, number of stimuli was systematically changed 1 to 10 . Conditioning-testing interval, $41 \mathrm{msec}$. In left graph, increment of antidromic action potential was plotted as percentage of control against number of conditioning stimuli. Samples of records on right side. C, control response. Others are conditioned by stimuli with number indicated in each record. Vertical bar, $100 \mu \mathrm{V}$. Horizontal bar, $1 \mathrm{msec}$.

In Fig. 8, a series of similar experiments was made on the geniculate evoked response. The presynaptic component of the response was depressed slightly by a single stimulus to the visual cortex (see record 1). Much more prominent depres- 

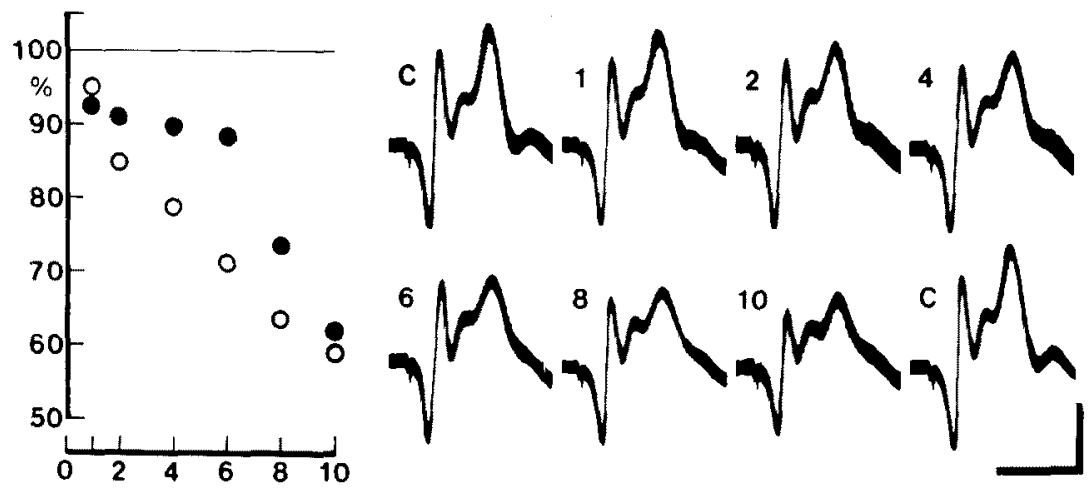

Fig. 8. Cortically induced depression of geniculate evoked response and number of stimuli to ipsilateral visual cortex. conditioning-testing interval, $80 \mathrm{msec}$. Parameter of conditioning stimulus to visual cortex was changed as in Fig. 7. Left graph, sizes of response plotted as percentage of control against number of stimuli. Filled and open circles refer to pre- and postysynaptic components, respectively. Right, sample of records. Vertical bar, $500 \mu \mathrm{V}$. Horizontal bar, 1 msec.

sion of the presynaptic component was caused when the number of stimuli was increased over six (see filled circles in the left graph). Similarly the depression of the postsynaptic component increased with the number of the conditioning stimuli. However, some discrepant behavior was observed between the two components; depression of the postsynaptic component as a function of the number of stimuli could be represented by a single curve, while that of the presynaptic one consisted of two parts.

Prolonged tetanic stimulation of the visual cortex

So far, the conditioning stimulus to the visual cortex was a brief tetanic one such as 5-10 pulses at $300 / \mathrm{sec}$. In this section, more prolonged repetitive stimulation as long as $2 \mathrm{sec}$ or more was used as a conditioning one and the effect on

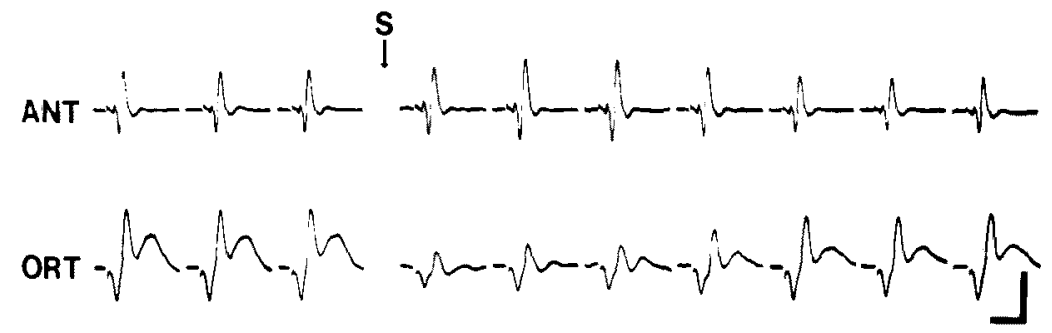

Fig. 9. Effect of prolonged tetanic stimulation of visual cortex on antidromic and orthodromic responses. These responses were obtained by procedure described in text. ANT, antidromic responses at optic tract. ORT, geniculate evoked responses. At mark $\mathrm{S}$, tetanic stimulation was applied to vistual cortex for 2 sec. Vertical bar, $500 \mu \mathrm{V}$. Horizontal bar, 1 msec. 
the geniculate evoked response and the antidromic action potential at the optic tract were examined. An example of the experiment is illustrated in Fig. 9. The antidromic action potential was evoked by a stimulus applied through the electrodes in the LGB and recorded with another pair of electrodes at the optic tract. Thus, the first record of the upper series was obtained. One second later, the orthodromic evoked response of the LGB was taken using the optic tract electrodes for stimulation and the LGB electrodes for recording (see the first record in the lower series). Thus, antidromic action potential and orthodromic evoked response were recorded alternately. At mark $S$, repetitive stimulation at a rate of $300 / \mathrm{sec}$ was applied to the ipsilateral visual cortex for $2 \mathrm{sec}$. This prolonged tetanic stimulation resulted in a marked increase of the antidromic action potential for about $10 \mathrm{sec}$ and in parallel with this increase, striking reduction of both presynaptic and postsynaptic components of the geniculate evoked response occurred.

\section{DISCUSSION}

The foregoing results showed that when conditioning was applied by a brief tetanus to the ipsilateral visual cortex, the presynaptic component of the geniculate evoked response was depressed for $300 \mathrm{msec}$ or more. The depression was always accompanied with increased excitability of the optic tract fibers close to their synaptic terminals. These findings indicate that the depression may be produced by a presynaptic process in the geniculate synapses. We should emphasize that in our experiments, neural connection between the retinal element and the optic nerve was completely blocked and therefore the indirect effect of cortical stimulation via the retinal element was not necessary to be taken into account. Another possibility to induce such depression may be presynaptic inhibition. ${ }^{7}$ This kind of inhibition is produced by the depolarization of the presynaptic terminals which in turn depresses the secretion of the transmitter substance to the postsynaptic membrane. The present experiments showed that the antidromic action potentials of submaximal size at the optic tract was augmented by cortical stimulation, suggesting that the augmentation was due to increased excitability of the presynaptic terminals (Fig. 5). On the other hand, the cortically induced depression was clearly observed even in the presynaptic component of the evoked geniculate response induced by supramaximal stimulation to the optic tract. This indicates that the depression is due to reduction in size of each action potential of the presynaptic fiber (Fig. 6). Since the increased excitability and the reduced amplitude of the action potential in the cathelectrotonic state of the nerve fiber is a well-established fact in the neurophysiology, it may well be assumed that the presynaptic terminals in the geniculate synapses are depolarized by repetitive stimulation of the visual cortex or its surroundings.

The concept of the presynaptic inhibition was extensively studied on the muscle afferent to the spinal cord and the secondary and tertiary neurons of the 
somesthetic system. However, no evidence of the presynaptic inhibition has been demonstrated in the LGB. Recently Szentágothai ${ }^{15}$ described axo-axonic contacts in the LGB and suggested that the structure might be the morphological basis of the presynaptic inhibition. Colonnier and Guillery ${ }^{6}$ also described a similar structure in the monkey LGB. Thus, our observation on the cortically induced depression of the geniculate evoked response must be a physiological counterpart of the anatomical structure. Iwama ${ }^{9}$ reached the same conclusion on the basis of his own physiological experiments. As to the pathway responsible for the inhibition, little information has been obtained. But it may be said that the effect is generated via polysynaptic pathways because repetitive stimulation is effective to produce the inhibition.

In recent investigations, it has been elucidated that visual information from the retina is not only relayed at the LGB, but also greatly controlled by extraretinal inputs to the nucleus., ${ }^{11,12}$ Stimulation of the midbrain reticular formation ${ }^{5,14,16}$ and its related subcortical structure ${ }^{13}$ give rise to great facilitatory effects on synaptic transmission in the LGB, while the striate and parastriate areas give facilitatory and/or inhibitory influence on the transmission. ${ }^{1,10,19}$ These extraretinal effects on the transmission can be detected by use of the evoked response of the LGB induced by optic tract stimulation as a measure of the number of the excited elements. Most previous authors have agreed that the main influence of the extra-retinal volleys is exerted on the postsynaptic component of the geniculate evoked response, and this fact suggests that the effect may be generated by a postsynaptic process. There are at least three possibilities as to the mechanism of the postsynaptic process under consideration, that is, i) a postsynaptic effect secondary to a presynaptic change, ii) after-hyperpolarization which may occur in the LGB neurons fired antidromically and iii) postsynaptic inhibition. So far as repetitive cortical stimulation is used, the depression of the postsyanptic component of the geniculate evoked response is clearly contaminated by the first possibility, because the presynaptic inhibition induced by such stimulation should be followed by corresponding reduction of synaptic transmission. If after-hyperpolarization exists, the depression of the postsynaptic component caused by ipsilateral cortical stimulation should be contaminated by the second possibility, ${ }_{17}^{17}$ but such a possibility is excluded in experiments with contralateral cortical stimulation. Therefore, the depression caused by contralateral cortical stimulation in Fig. $1 \mathrm{E}$ in which the presynaptic component remained almost unaltered, must be due to postsynaptic inhibition.

\section{Acknowledǵment}

The authors wish to thank Dr. K. Motokawa for his invaluable discussion and suggestion throughout the course of the experiment and the preparation of the manuscript. Thanks are also due to Mr. S. Sai for his technical assistance. 


\section{References}

1) Ajmone-Marsan, C. \& Morillo, A. Electroenceph. clin. Neurophysiol., 1961, 13, 553.

2) Andersen, P., Eccles, J.C., Schmidt, R.F. \& Yokota, T. J. Neurophysiol., 1964, 27, 92.

3) Bishop, P.O., Jeremy, D. \& Lance, J.W. J. Physiol., 1953, 121, 415.

4) Bishop, P.O. \& McLeod, J.G. J. Neurophysiol., 1954, 17, 387.

5) Bremer, J. Sensory Communication, edited by W.A. Rosenblith, the M.I.T. Press, Cambridge, 1961.

6) Colonnier, M. \& Guillery, R.W. Zschr. Zellforsch., 1964, 62, 333.

7) Eceles, J.C. Physiology of Synapses, Springer, Berlin, 1964.

8) Eceles, J.C., Eccles, R.M. \& Magni, F. J. Physiol., 1961, 159, 147.

9) Iwama, K., Sakakura, H. \& Kasamatsu, T. Jap. J. Physiol, 1965, 15, 310.

10) Kwak, R. Tohoku J. exp. Med., 1965, 86. 290.

11) Motokawa, K. Int. Rev. Neurobiol., 1963, 5, 121.

12) Motokawa, K. \& Suzuki, H. in Progress in Brain Research, edited by P. Schadé, Elsevier, Amsterdam, in press.

13) Okuda, J. Tohoku J. exp. Med., 1962, 76, 350.

14) Suzuki, H. \& Taira, N. Jap. J. Physiol., 1961, 11, 641.

15) Szentágothai, J. Information Processing in the Nervous System, edited by R.W. Gerard, Excerpta medica foundation, Amsterdam, 1964.

16) Taira, N. \& Okuda, J. Tohoku J. exp. Med., 1962, 78, 76.

17) Vastola, E.F. J. Neurophysiol., 1959, 22, 258.

18) Wall, P.D. \& Johnson, A.R. ibid., 1958, 21, 148.

19) Widén, L. \& Ajmone-Marsan, C. Exp. Neurol., 1960, 2, 468. 